(2) Open Access Full Text Article

\title{
Malignant mixed ovarian germ cell tumor composed of immature teratoma, yolk sac tumor and embryonal carcinoma harboring an EGFR mutation: a case report
}

This article was published in the following Dove Press journal: OncoTargets and Therapy

\section{Yao Wang \\ Jia-Xin Yang \\ Mei Yu \\ Dong-Yan Cao \\ Keng Shen}

Department of Obstetrics and Gynecology, Peking Union Medical College Hospital, Chinese Academy of Medical Sciences and Peking Union Medical College, Beijing, China
Correspondence: Jia-Xin Yang Department of Obstetrics and Gynecology, Peking Union Medical College Hospital, Chinese Academy of Medical Sciences and Peking Union Medical College, No I Shuaifuyuan, Dongcheng District, Beijing 100730, China

Tel +861069154022

Fax +861069155635

Email yangjiaxin_2017@I63.com

\begin{abstract}
Malignant mixed ovarian germ cell tumors are very rare, accounting for $\sim 5.3 \%$ of all malignant ovarian germ cell tumors (MOGCTs), with a very high degree of malignancy. The treatment of patients with persistent, refractory, or platinum-resistant MOGCT is not well defined. The objective of this case report was to analyze the importance of chemotherapy, salvage surgery and target therapy in the treatment of a patient with refractory OGCT after first-line chemotherapy failure. We reported a 34 year-old woman suffered from advanced refractory MOGCT after first-line chemotherapy, cytoreductive surgery, and a series of chemotherapy. The genetic test shows she is a carrier of EGFR: p.L858R mutation. Based on genetic testing result, she received icotinib which targeted for EGFR mutation, but the tumor progressed. After a secondary cytoreductive surgery, she exhibited a partial response and continued to receive chemotherapy. This suggests that salvage surgery may be considered for patients with persistent or refractory MOGCTs when no effective systemic treatment option is available. Targeted therapies based on gene sequencing may provide a new option; however, its efficacy and related resistance mechanisms still need to be verified by further study.
\end{abstract}

Keywords: mixed ovarian germ cell tumor, next-generation sequencing, EGFR mutation, target therapy, salvage surgery

\section{Introduction}

Malignant ovarian germ cell tumors (MOGCTs) are rare but aggressive tumors in the female genital system, and primarily affect adolescents and young adults. ${ }^{1}$ MOGCTs represents $2 \%-3 \%$ of all ovarian malignancies in the Western countries, whereas they may account for $\sim 15 \%$ of ovarian cancers in Asians and in blacks. ${ }^{2}$ However, Surveillance, Epidemiology, and End Results (SEER) data (1972-2002) suggested lower incidence rates in blacks than whites and other nonwhites, and higher incidence rates for Asian/Pacific Islanders and Hispanics. ${ }^{3}$ Since these tumors mainly occur in women of reproductive age, they are usually diagnosed during pregnancy. ${ }^{4}$ Although MOGCTs are highly aggressive, they can be cured by surgery and chemotherapy. PEB (cisplatin, etoposide, and bleomycin) chemotherapy is the recommended first-line chemotherapy regimen. The cure rates for patients with early-stage MOGCT approach $100 \%$, and even in advanced-stage disease are at least $75 \% .^{5}$ In our previous study, the 5-year disease-free survival (DFS) for all MOGCTs patients was $86.00 \%$, and the 5-year overall survival (OS) was predicted to be $95 \% .^{2}$ Although most patients with 
MOGCT can achieve complete cure by the combination of surgery and chemotherapy, a small percentage do have persistent or recurrent disease. ${ }^{5}$ For patients with persistent, refractory or platinum-resistant (relapse within 6 weeks of completing platinum-based chemotherapy) recurrent tumor, the prognosis is much worse..$^{5,6}$

Germ cell tumors (GCTs) of ovary and testis share many similarities in morphology and chromosomal alterations. ${ }^{7}$ Comprehensive genome sequencing in testicular and mediastinal GCTs have identified that the primary somatic feature of GCTs is highly recurrent chromosome arm level amplifications and reciprocal deletions (reciprocal loss of heterozygosity), variations that are significantly enriched in GCTs. Subsets of these tumors also acquire KRAS-activating mutations. ${ }^{8}$ However, genomic features in ovarian germ cell tumors (OGCTs) that contribute to the chemoresistance and progression remain incompletely characterized. Limited previous studies have identified some mutations in OGCTs, such as BRCA1/2, KIT, MAPK1, and DICER1. ${ }^{9-14}$ Nevertheless, the application and efficacy of target therapy has never been described.

Epidermal growth factor receptor (EGFR, also known as ERBB1 or HER1) is the most common driving gene in nonsmall-cell lung cancer. Front-line epidermal growth factor receptor tyrosine kinase inhibitor (EGFR TKI) therapy is the standard care for lung cancer patients with EGFR mutations (exon 19 deletion or L858R mutation). ${ }^{15}$ Recently, EGFR has been shown to be overexpressed in many human cancers, including lung, central nervous system, head and neck, bladder, pancreas, breast, and uterine corpus; and it correlates with poor prognosis. ${ }^{16}$ Therapies targeting EGFR signaling pathway are part of the arsenal of agents that are used to treat lung, colorectal, pancreatic, and head and neck cancers. ${ }^{17}$ To the best of our knowledge, there have been no reports of EGFR mutation in MOGCT so far. Here, we report a case of platinum-resistant mixed MOGCT harboring a previously unreported EGFR somatic mutation for the first time.

\section{Case report}

The patient is a 34-year-old woman diagnosed with ovarian mixed germ cell tumor after cesarean section. On February 17, 2017, her ultrasonography at 36 weeks' gestation showed a complex solid and cystic mass $(18 \times 2 \times 1.5 \mathrm{~cm})$ located between spleen and the anterior inferior part of left kidney. No abnormalities were found during the period of previous routine pregnancy check-ups. She had no family history of gynecologic or other malignancies. Tumor marker evaluation showed serum carbohydrate antigen 125 (CA125) and alpha fetoprotein (AFP) were elevated (CA125 $702 \mathrm{U} / \mathrm{mL}$; AFP $174 \mathrm{ng} / \mathrm{mL}$ ). She subsequently underwent cesarean section, left salping-oophenrectomy, and multiple biopsy of peritoneum and great omentum in other hospital. According to the surgical record description, there were $1,000 \mathrm{~mL}$ bloody ascites in the abdominal cavity. Intraoperatively, the mass was found to originate from the left ovary, and tightly adhere to surrounding bowel and pelvic wall. The parietal peritoneum was covered with miliary hard nodules. Postoperative pathology showed ovarian yolk sac tumor (YST) with allogeneic cartilage tissues. Omentum and peritoneum were free of tumor. Immunohistochemical study showed diffusely positive staining for SOX2 (Sry-related HMG box2), OCT3/4 (octamer-binding transcription factor 3/4), and SALL-4 (sallike protein 4), focally positive for Syn (synapsin), AE1/AE3, CK7 (cytokeratin 7), and GATA-3 (GATA binding protein 3), weakly positive for AFP and negative for CD117 (cluster of differentiation), $\beta$-HCG, GFAP (glial fibrillary acidic protein), and EMA (epithelial membrane antigen).

After recovery from the surgery, the patient visited our hospital. A whole-body positron emission tomographycomputed tomography (PET-CT) revealed multiple metastases in the greater omentum, pelvic cavity, intestine surface, subcapsular of the liver, mediastinum, cardio phrenic angle, right internal mammary node and subclavian lymph node, and the largest metastases located in the left middle abdomen and next to the descending colon (sizes are $3.9 \times 5.8 \times 5.8 \mathrm{~cm}$ and $2.8 \times 6.1 \times 7.2 \mathrm{~cm}$, respectively) (Figure 1A). Serum levels of AFP, CA125 and $\beta$-HCG were elevated at $87.6 \mathrm{ng} / \mathrm{mL}, 187.7 \mathrm{U} / \mathrm{mL}$ and $101.3 \mathrm{IU} / \mathrm{L}$. In addition, an expert comprehensive pathology review sample from the initial surgery was undertaken, and the result showed a primordial GCT, consisting of G2 immature teratoma (IT), YST, and embryonal carcinoma.

After the new diagnosis, she received 1 cycle of PEB (cisplatin, etoposide, and bleomycin, 3-day regimen) with tumor progression indicated by image and tumor markers. After a discussion of the gynecologic oncology professional group, considering the embryonal carcinoma component in the tumor, the regimen was diverted to the EMA/CO (etoposide, methotrexate, actomycin D, cyclophosphamide, and vincristine) regimen which was commonly used in gestational trophoblastic neoplasia (GTN), and resulted in a slow decrease in $\beta$-HCG and CA125 but an increase in AFP level. The therapeutic efficacy was not satisfactory, and the patient subsequently underwent cytoreductive surgery (total hysterectomy, right salping-oophenrectomy, omentectomy, mesenteric, and retroperitoneal tumor resection). She was 

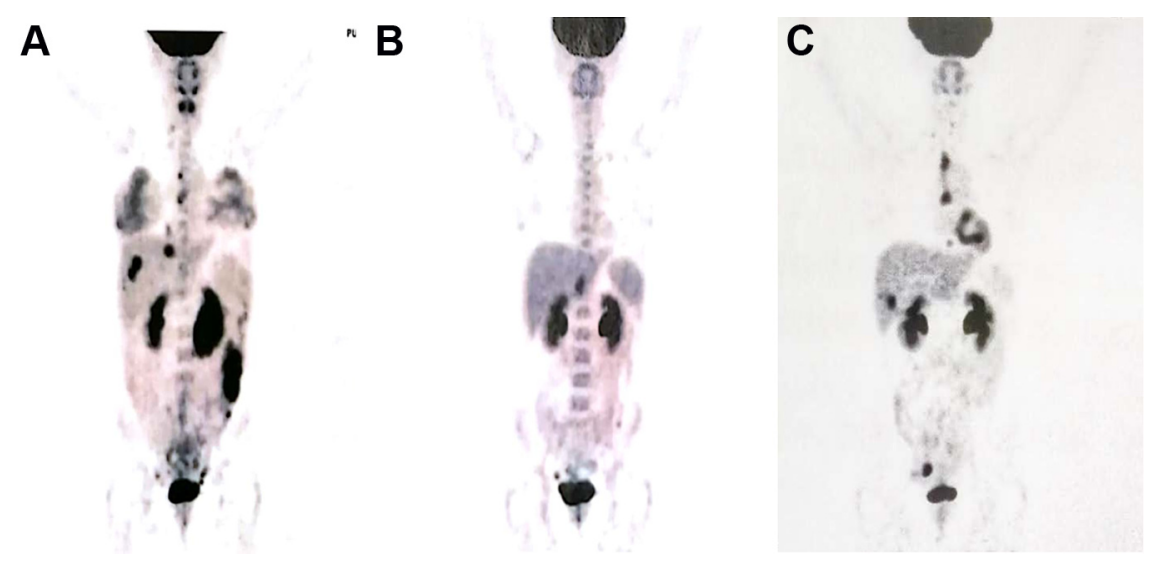

Figure I Changes of residual lesions showed by PET-CT.

Notes: (A) March 10, 2017, before chemotherapy and cytoreductive surgery; (B) September 19 2017, after cytoreductive surgery and nine courses of chemotherapy; (C) April 4, 2018, before secondary cytoreductive surgery.

Abbreviation: PET-CT, positron emission tomography-computed tomography.

noted to have extensive pelvic and abdominal carcinomatosis, multiple military tumor nodules scattering on omentum, small intestine, descending colon, and rectum, left abdominal wall, the descending colonic sulcus, pelvic peritoneum, and bladder peritoneal reflex. The larger metastases were found in the mesentery and retroperitoneum. Debulking was optimal. The gross lesions were basically resected (Figure 2A and B), leaving only a small amount of miliary residues on the surface of the peritoneum. Changes of residual lesions by cytoreductive surgery are shown in Figure 3. Postoperative pathology (Figure 4) proved the tumor was a MOGCT with G2 IT, small amount of YST and embryonal carcinoma. The uterus and right accessory were free of tumor. The immunohistochemical profile showed positive staining for SOX2 (embryonal carcinoma component), OCT3/4 (embryonal carcinoma component), and SALL-4 (IT, YST and embryonal carcinoma component), AFP (IT, YST and embryonal carcinoma component), AE1/AE3 (YST and embryonal carcinoma component), CK7 (YST and embryonal carcinoma component) and negative for GPC-3 (glypican-3) (embryonal carcinoma) and GATA-3. Postoperatively, she commenced on 2 cycles of PEB (5-day regimen) chemotherapy which resulted in decreased AFP but increased $\beta$-HCG level. Therefore, the regimen was switched to EMA/EP (etoposide, methotrexate, and actinomycin D/etoposide and cisplatin) chemotherapy which aimed at high risk and drug resistant GTN. Interestingly, an opposite changing trend of tumor markers was observed during EMA/EP chemotherapy. Due to the severe bone marrow suppression, the chemotherapy was changed to PVB (cisplatin, vincristine, bleomycin, 3-day regimen) chemotherapy which reduced the toxicity of myelosuppression. During this cycle, the serum level of AFP and
$\beta$-HCG were both increased. Considering the progression of the tumor, from July 20, the chemotherapy was changed to PEB and bevacizumab for three courses, encouragingly the level of $\beta$-HCG and AFP both had fluctuant reduction, but the reduction of AFP was not obvious.

After three cycles of PEB and bevacizumab chemotherapy, PET-CT (Figure 1B) showed resolution of her previous disease burden but a new metastasis emerged on the caudate lobe of the liver. In September, the chemotherapy was changed to TIP (paclitaxel, ifosfamide, and cisplatin) followed by 1 cycle of TIP and bevacizumab chemotherapy. Due to the intolerable neurotoxicity of paclitaxel, the regimen was then changed to DIP (docetaxel, ifosfamide and cisplatin) plus bevacizumab chemotherapy (6-day regimen) for 3 cycles. During the last three cycles, there was a fluctuant reduction in $\beta$-HCG level, but the level of AFP slowly increased. The main side effects of chemotherapy were bone marrow IV degree depression and fatigue. Considering drug resistance of liver metastases, the patient underwent CTguided radiofrequency ablation (RFA) of liver metastases. Unfortunately, the serum level of AFP and $\beta$-HCG gradually increased. At the patient's request and considering the suboptimal responsive of chemotherapy, a whole exme sequencing (WES) was performed and an EGFR somatic mutation (p.L858R) was identified (Figure 5). This suggests that this patient may benefit from EGFR TKI, which includes erlotinib, gefitinib, icotinib, and afatinib. After full discussion with the patient, she decided to receive icotinib (the first generation of EGFR TKI) treatment. Unfortunately, results of examination a week later showed elevated tumor markers (AFP 117.7 ng/mL, $\beta$-HCG 3349 IU/L). A CT scan (Figure 6) and PET-CT (Figure 1C) both revealed multiple 

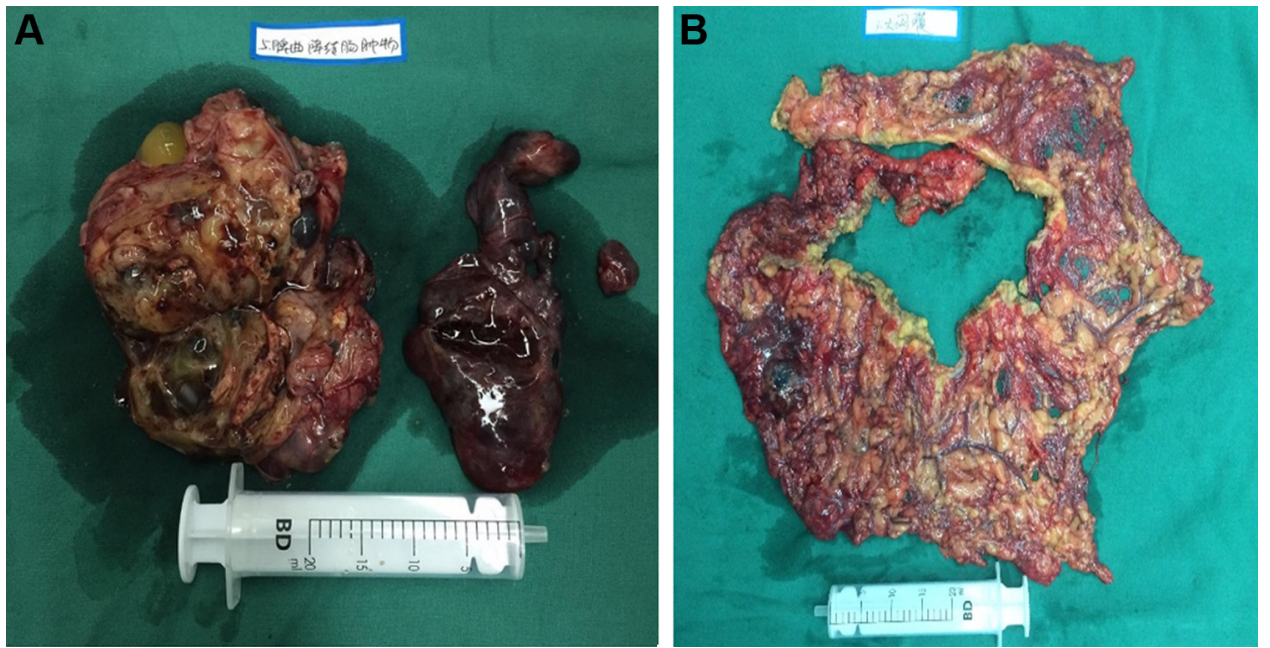

Figure 2 Some specimens at first cytoreductive surgery.

Notes: (A) Metastases located between spleen and descending colon; (B) omentum.
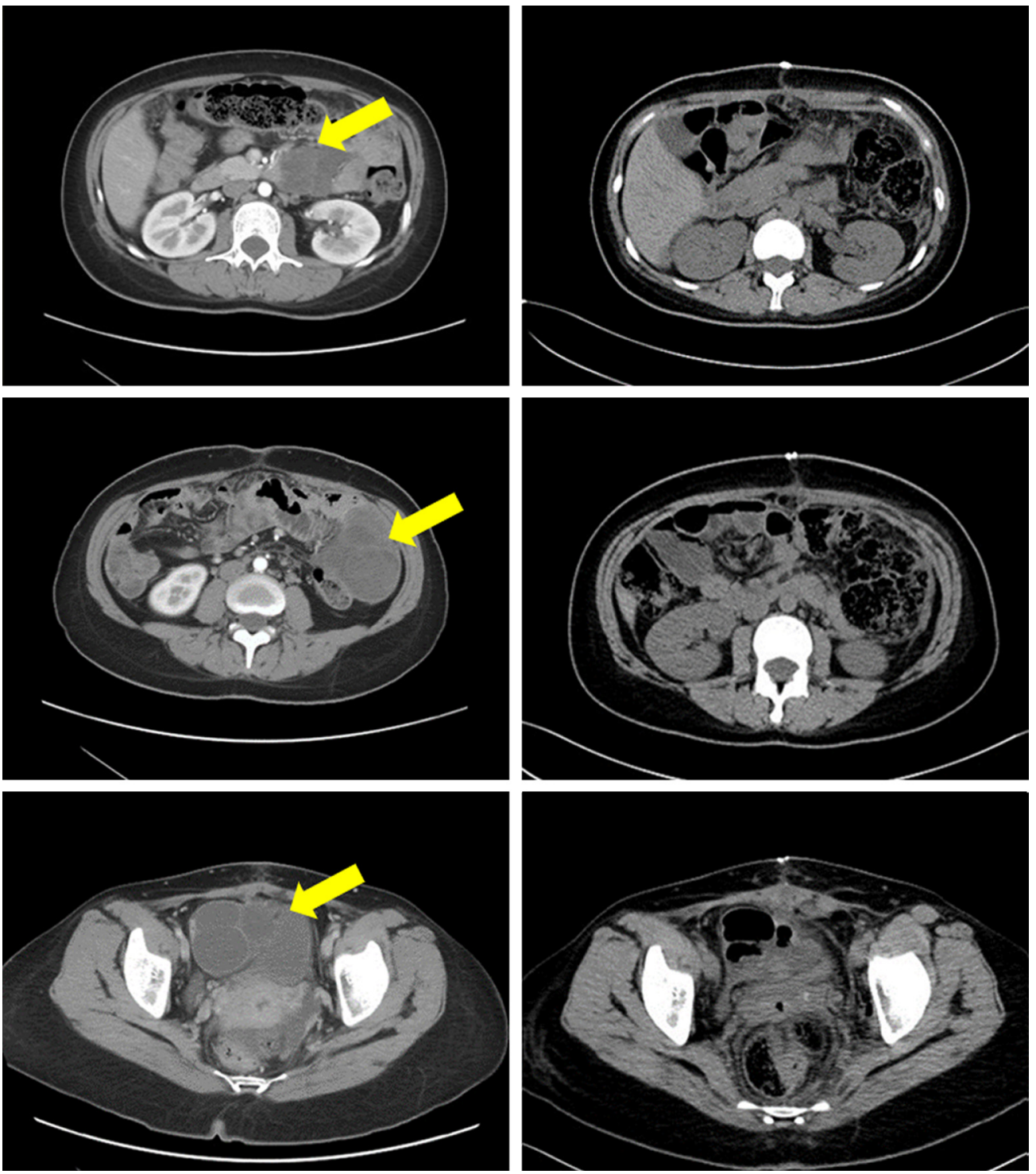

Figure 3 Changes of residual lesions by cytoreductive surgery.

Notes: The yellow arrows represent metastases. Most metastases disappeared after cytoreductive surgery (left: before cytoreductive surgery; right: after cytoreductive surgery). 

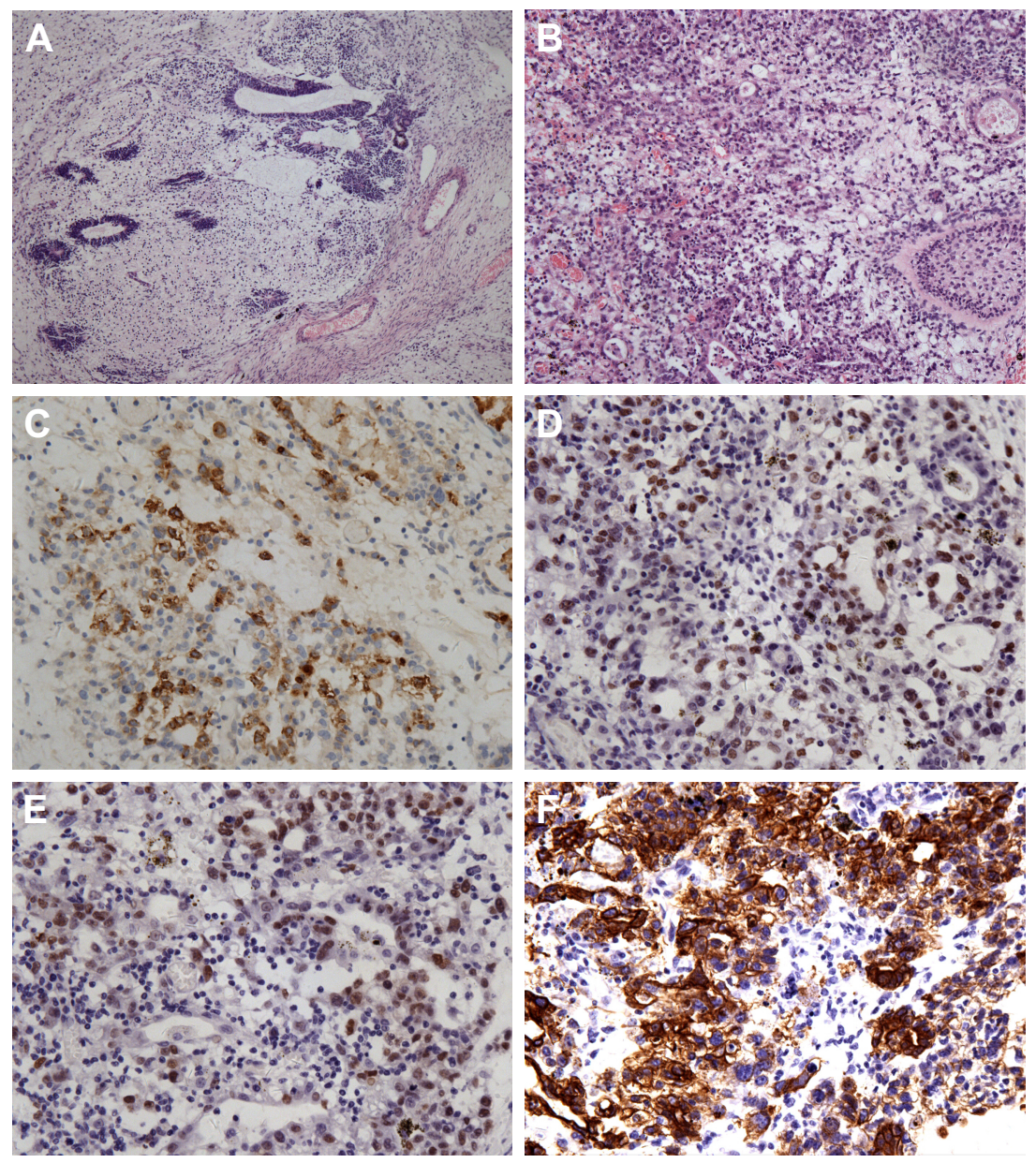

Figure 4 Postoperative pathology proved the tumor was a MOGCT with immature teratoma (G2), small amount of YST and embryonal carcinoma.

Notes: (A) immature teratoma component (HE, I00×); (B) YST and embryonal carcinoma component (HE, 400×); (C) AFP immunostain diffusely positive in YST component (400×); (D) SALL-4 positive in YST and embryonal carcinoma component; (E) SOX2 positive in embryonal carcinoma; (F) AEI/AE3 positive in YST and embryonal carcinoma component.

Abbreviations: MOGCT, malignant ovarian germ cell tumor; YST, yolk sac tumor.

metastases around the whole body. Subsequently, she underwent the secondary cytoreductive surgery involving resection of metastases on liver surface, hepato-diaphragmatic space, hepato-renal space, peritoneal surface, intestinal surface, right obturator, left rib margin extraperitoneal metastasis, and right iliac artery lymph node. Metastases between liver and diaphragm are shown in Figure 7. Tumor markers decreased significantly after surgery. The patient recovered well and received PEB chemotherapy on the 6th postoperative day. After chemotherapy, the level of AFP decreased to normal range for the first time but the $\beta-H C G$ continued to rise. Given the elevated $\beta$-HCG level, the chemotherapy regimen was changed to EMA/EP. Due to the impaired renal function, regimen was changed to FAEV (FUDR, actinomycin $\mathrm{D}$, etoposide, and vincristine) which alleviated the toxicity to the kidney but was also an effective regimen for high risk and drug resistant GTN. However, this regimen was ineffective. Considering the side effects containing myelosuppression, impaired renal function and neurotoxicity on this patient, the patient was treated with docetaxel and gemcitabine from July 5, 2017. The efficacy evaluation was partial response. She is going to receive the next course of chemotherapy. Changes in tumor markers during the whole course of the disease was shown in Figure 8.

\section{Discussion}

This case is important to report for several reasons. First, it was a rare case of mixed GCTs with components of YST, IT and embryonal carcinoma which is the least common component of GCTs. Second, the routine pregnancy check-up before 36 weeks of gestation was normal, which indicated some tumors may be neglected during pregnancy. Third, this type of tumor exhibited primary resistance to first-line regimen of GCT. Different components of the tumor showed different therapeutic response to chemotherapy regimen. Fourth, the patient harbors the common EGFR-activating mutation 


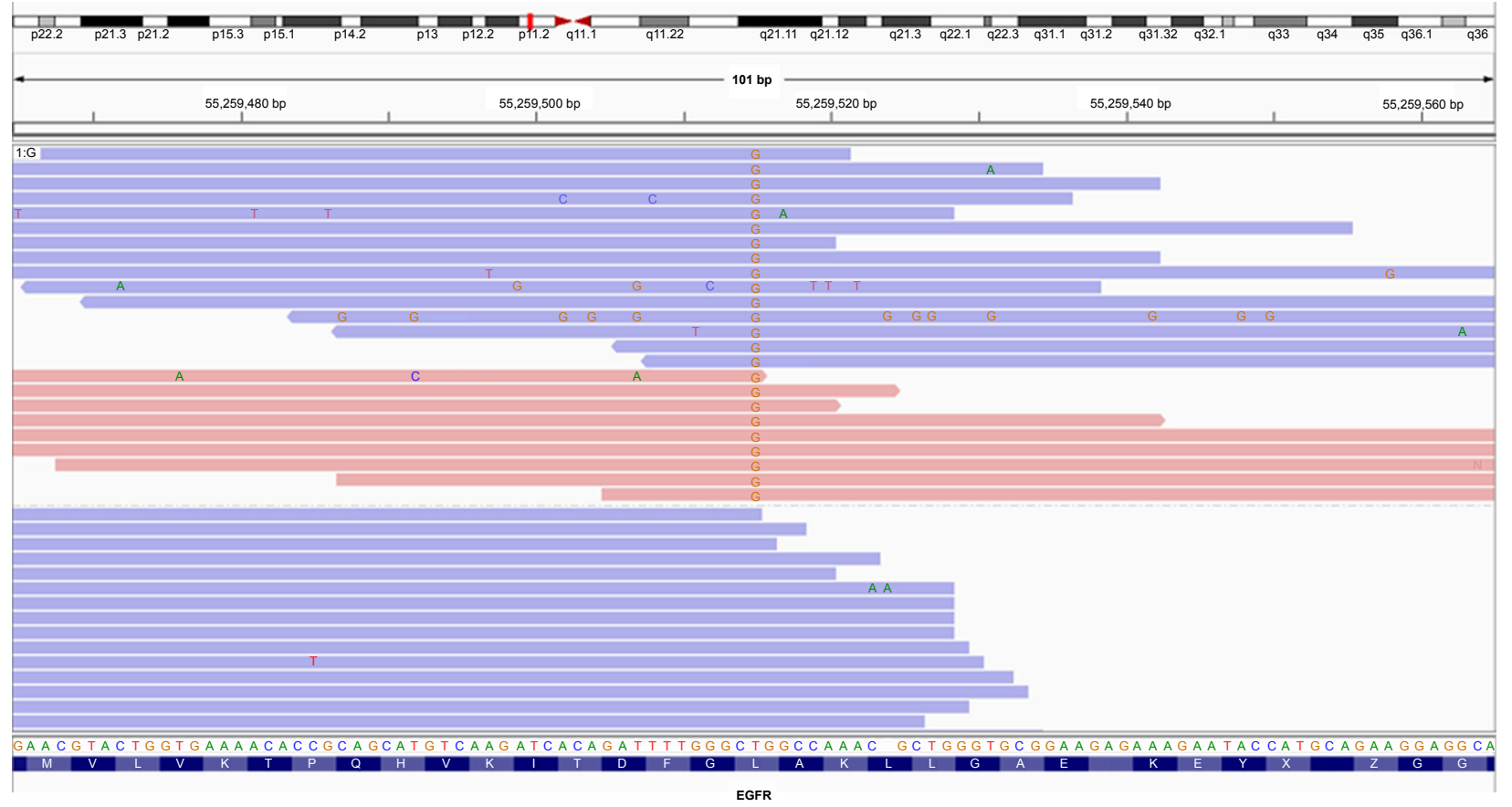

Figure $\mathbf{5}$ The result of comprehensive genomic profiling revealed an EGFR somatic mutation (p.L858R).

(L858R) which usually predicts sensitivity to EGFR TKIs, but the patient did not show response to EGFR TKI.

MOGCTs are heterogeneous tumors that are derived from the primitive germ cells of the embryonic gonad, accounting for about $2.6 \%$ of all ovarian malignancies. ${ }^{18}$ MOGCTs mainly manifest in adolescence, usually with characteristic elevated serum tumor marker levels, which may aid in diagnosis, evaluation of therapy, and post-treatment surveillance. Mixed ovarian germ cell tumors are composed of at least two germ cell components. Some types of malignant elements, such as YST, high-grade IT and embryonal carcinoma, are associated with a more aggressive behavior. ${ }^{19}$ The tumor of
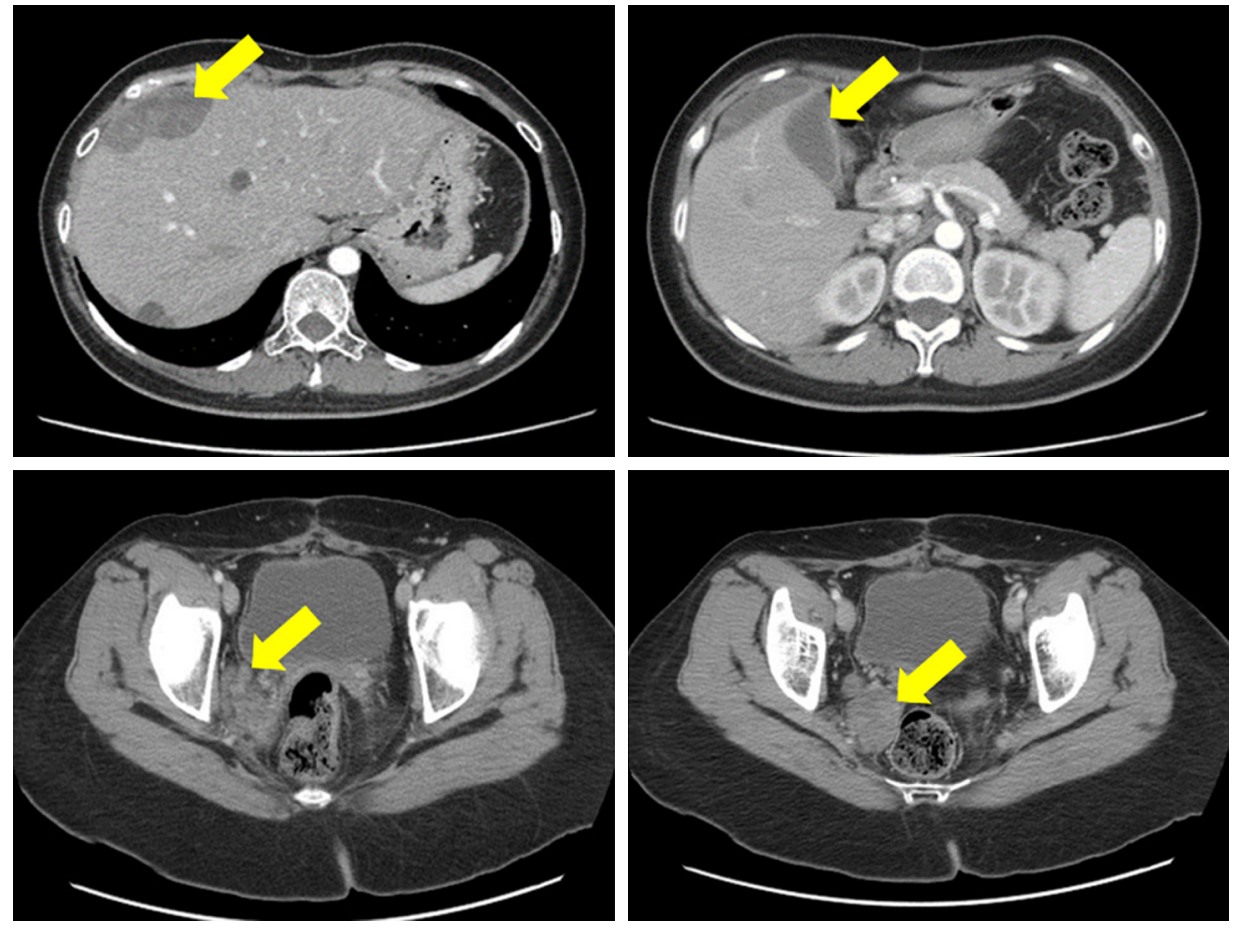

Figure 6 CT scan revealed multiple metastases.

Notes: The yellow arrows represent metastases. Metastases were mainly located in the subcapsular area of the liver and in the right attachment area. 

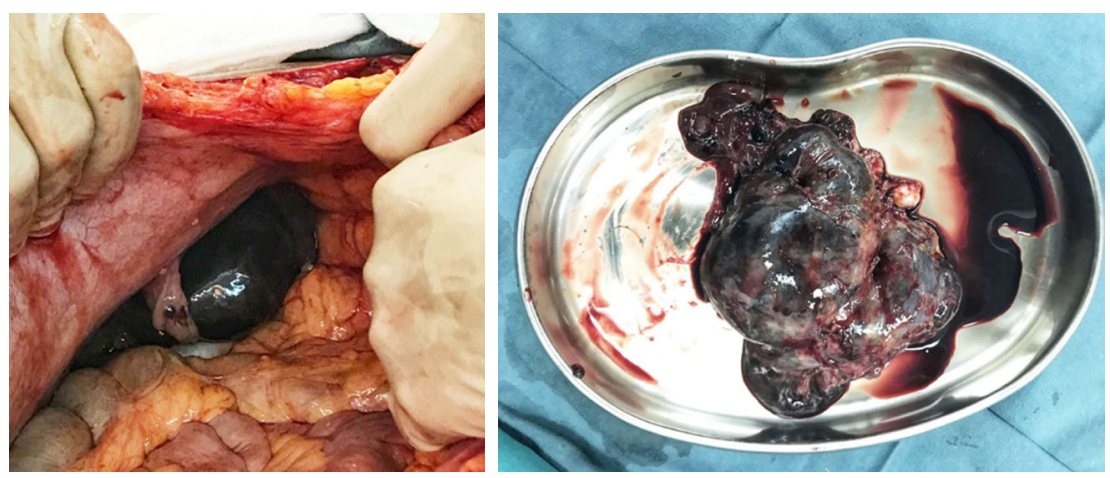

Figure 7 Metastases between liver and diaphragm.

this patient was composed of 3 histological components, of which the YST and embryonal carcinoma components have been suggested as high-risk features, and usually result in poor prognosis. ${ }^{20}$ Serum AFP and $\beta$-HCG were reliable and sensitive biomarkers for MOGCTs, and elevation of both $\mathrm{AFP}$ and $\mathrm{hCG}$ levels is also a strong predictor of poor survival shown in previous analysis. ${ }^{20-22}$ Therefore, we monitored the levels of serum AFP and $\beta$-HCG to indicate the therapeutic effect in this case.

The standard management of MOGCTs is complete surgical excision. Based on tumor stage and histological type, some patients need to receive chemotherapy after surgery. Fertility-sparing surgery can be performed for patients regardless of tumor stage, because the majority are unilateral and highly sensitive to chemotherapy. Furthermore, neither comprehensive staging nor aggressive debulking improves the patients' survival. ${ }^{2,23}$ Due to the rarity of advanced stage MOGCTs, the role of neoadjuvant chemotherapy (NACT) in MOGCTs is not well defined. ${ }^{24}$ According to the retrospective study conducted in our center, we observed that patients treated with NACT showed less peri-operative morbidity and better optimal cytoreduction rate. ${ }^{25}$ As for this case, NACT was commenced because of bulky tumor burden, but the patient did not show response to NACT, so she immediately received interval debulking surgery and adjuvant chemotherapy.

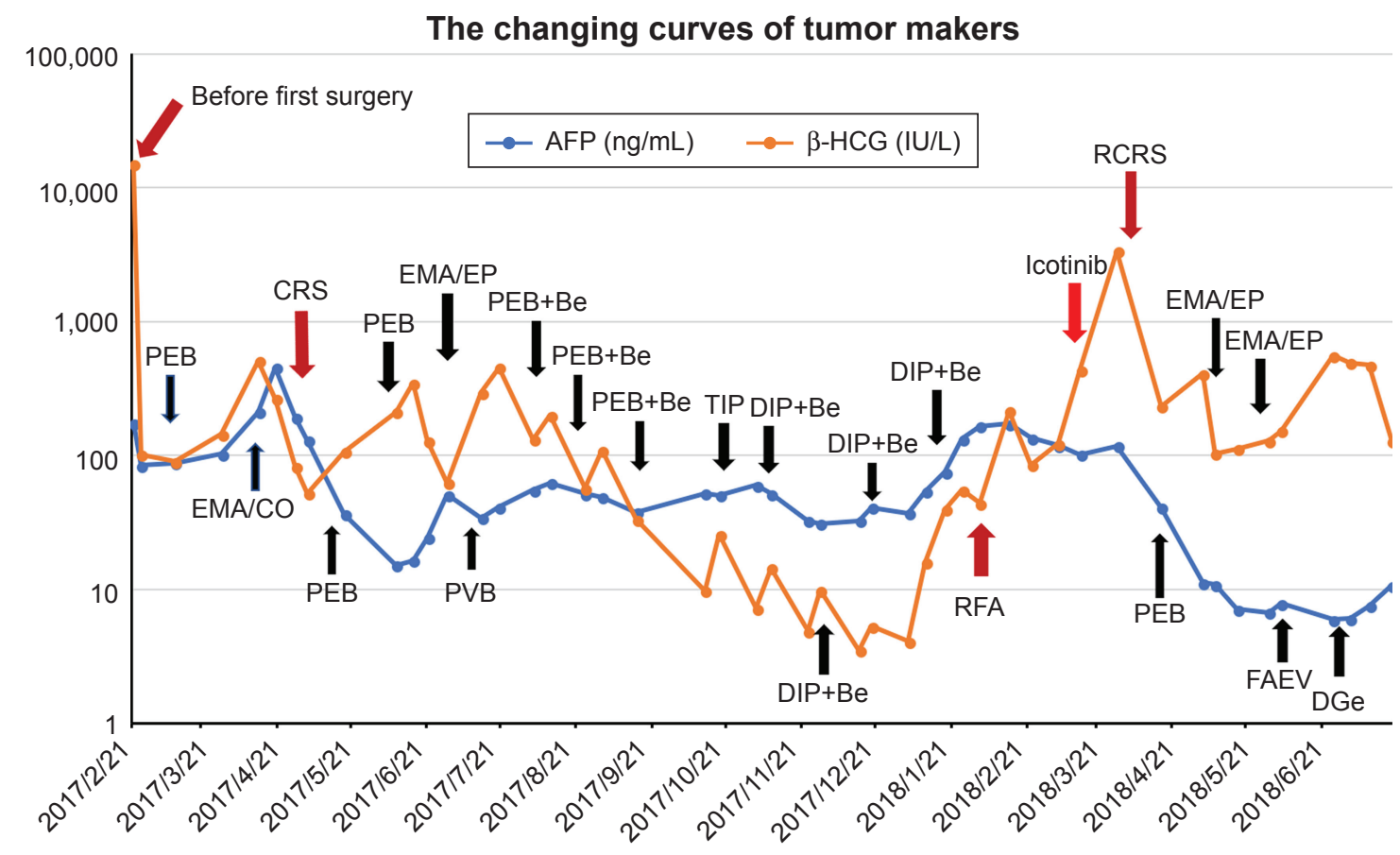

Figure 8 Serum AFP and $\beta$-HCG levels during treatment.

Abbreviation: A, actinomycin D; AFP, alpha fetoprotein; B, bleomycin; Be, bevacizumab; C, cyclophosphamide; CRS, cytoreductive surgery; D, docetaxel; E, etoposide; F, FUDR; Ge, gemcitabine; I, ifosfamide; M, methotrexate; P, cisplatin; RCRS, re-cytoreductive surgery; RFA, radiofrequency ablation; T, taxol; $V$, vincristine. 
The evolution in the chemotherapeutic treatment of MOGCTs constitutes a major advance in clinical management. ${ }^{5}$ PEB regimen has become the standard chemotherapy regimen for patients with MOGCT since 1990 and remains so to the present. ${ }^{26,27}$ Most patients can be cured by surgery and first-line chemotherapy, but $\sim 20 \%$ of patients had recurrent or refractory disease. ${ }^{2,20}$ Only $10 \%$ of patients treated with standard-dose salvage chemotherapy achieved long-term survival. ${ }^{20}$ The particularity of this case was that the tumor consisted of three histological types, and different histological components showed different sensitivities to chemotherapy which were reflected by levels of tumor markers. Therefore, we need to consider the chemotherapysensitive regimen of each component when selecting a chemotherapy regimen. Based on the elevated $\beta$-HCG level, we selected alternative regimens commonly used to treat high-risk GTN which was also characterized by elevated serum $\beta$-HCG level.

Because of the rarity of relapse and drug resistance in this population, there is no standard approach and the available treatment strategies currently all come from treatment experience of testicular cancer. ${ }^{28-31}$ TIP regimen and/or high-dose chemotherapy (HD-CT) with hematopoietic stem cell rescue is an effective second-line therapy for patients with relapsed testicular GCTs. Kondagunta et a ${ }^{28}$ observed a durable complete response (CR) rate in relapsed testicular GCT patients treated with TIP as second-line therapy. In a retrospective review of 184 consecutive patients conducted by Einhorn et al, ${ }^{31}$ patients with metastatic testicular cancer who had progressed after receiving cisplatin-based combination chemotherapy regimens were proved to be potentially curable by means of HD-CT plus hematopoietic stem-cell rescue, even when this regimen is used as third-line or later therapy or in patients with platinum-refractory disease. Although HD-CT with stem-cell rescue appears to be favored for patients with persistent, refractory, or platinum-resistant recurrent MOGCTs, the salvage rate appears to be no better than 50\%. ${ }^{5}$ Reddy Ammakkanaver et al ${ }^{32}$ reported 13 patients who received HD-CT for recurrent MOGCT from 1990 to 2013. Median survival was 11 months (range 4-270 months). Seven patients achieved CR, including all five patients with platinum-sensitive disease. Four of these seven patients achieving $\mathrm{CR}$ remain disease free (continuously no evidence of disease) at 12, 22, 120, and 270 months after receiving only HD-CT. In 2017, Giorgi et $\mathrm{al}^{33}$ conducted a retrospective analysis of female patients with GCT treated with HD-CT registered with the European Society for Blood and Marrow Transplantation (EBMT). In total, 25 of 60 patients (42\%) were progression-free following HD-CT at a median follow-up of 87 months (range 3-219 months). This provided evidence that patients with recurrent female GCT can achieve long-term DFS and cure potential with salvage HD-CT. ${ }^{33}$ And this represents the largest data set evaluating outcomes of salvage HD-CT in MOGCT. In this case, the efficacy of TIP or DIP was not satisfactory. During the first 4 cycles of TIP or DIP, there was an obvious decrease in $\beta$-HCG level, while level of $\beta$-HCG and AFP started to increase from the fifth cycle which suggested acquired drug resistance. In patients where no systemic treatment option is available or no chemotherapy regimen can be applied to, salvage surgery approaches may be an option in highly selected cases, which is then called desperation surgery. ${ }^{34}$ In retrospective analysis, long-term DFS rates of $21 \%-50 \%$ were documented. ${ }^{35-37}$ This patient received a second cytoreductive surgery because of drug resistance related poor tumor control and resectable metastatic lesions. The effect of salvage surgery was significant and the tumor markers were significantly reduced. It should be noted that serum AFP level was under control by salvage surgery and the following chemotherapy, which implied that patients with an elevated AFP are more likely to benefit from combination of cytoreductive surgery and following chemotherapy. However, when there was embryonal component with elevated $\beta$-HCG level, the surgery seemed less successful, especially when there were no effective chemotherapy regimens available.

Given the relatively low response rates of these salvage therapies, novel treatment approaches are urgently needed to improve outcomes of patients with chemo-refractory, multiply relapsed GCTs. Studies aimed to investigate the efficacy of immunotherapy that targets the PD-1/PD-L1 pathways in GCTs have been conducted. Two studies showed that testicular GCTs expressed higher levels of PD-L1 than normal testicular tissues. ${ }^{34,35}$ Adra et $\mathrm{al}^{38}$ first reported the efficacy of immune checkpoint inhibitors in GCT. But the result showed pembrolizumab does not appear to have clinically meaningful single-agent activity in refractory GCT. Whether or not PD-L1 can be used as a reliable biomarker for predicting response to immunotherapy still needs to be elucidated in future trials.

The application of genetic sequencing of tumors has found specific mutations in certain signaling pathways in a variety of cancers, which can guide us to find the corresponding targeted drug for individualized treatment. This has led to a shift in cancer treatment from non-selective cytotoxic drugs to targeted therapies. ${ }^{39}$ To date, the detailed 
molecular aberrations underlying mixed MOGCTs remain largely unknown. A novel EGFR p.L858R somatic mutation was detected in the presented case. EGFR, which belongs to the ERBB family (EGFR/HER/ERBB) of receptor tyrosine kinases, is known to contribute to cell survival signals as well as metastatic potential of some tumors, including epithelial ovarian cancer. ${ }^{39,40}$ However, there is no relevant research in MOGCT with EGFR mutation. The efficacy of the first-line EGFR inhibitors gefitinib and erlotinib over cytotoxic chemotherapy in patients with EGFR-mutant NCSLC has been well established. It is now appreciated that somatic ERBB receptor mutations occur at relatively low frequencies across multiple tumor types. ${ }^{41}$ Clinical studies are underway to determine whether tumors harboring these alterations respond to small molecule EGFR inhibitors. To date, anti-EGFR antibody, anti-EGFR, or dual EGFR/HER2 tyrosine kinase inhibitors have been evaluated across a variety of disease types. ${ }^{16,42}$ Clinical trials of EGFR-targeted therapeutics for the treatment of ovarian cancer over the past decade have yielded only modest results to date. ${ }^{40}$ Recent evidence suggests that some somatic ERBB receptor mutations render resistance to FDAapproved EGFR inhibitors. ${ }^{42,43}$ Researchers implies reasons for resistance to EGFR targeted therapy can be grouped to the following categories: mutation of EGFR to a drug-resistant state; 'oncogenic shift,' or activation of a bypass signaling pathway; impairment of a pathway that is essential for EGFR TKI-mediated apoptosis, and histologic transformation to small cell lung cancer or an epithelial-mesenchymal transition. ${ }^{17}$ All four resistance mechanisms have been observed in lung adenocarcinoma with resistance to EGRF TKIs, ${ }^{44}$ and which kind of mechanisms is responsible for the resistance to EGFR TKI in this case needs further research to explore.

In our case, the patient with refractory MOGCT had primary drug resistant to standard chemotherapy regimen. Although an EGFR mutation was detected in the samples of this patient, she did not show response to the target therapy. A salvage surgery was performed and achieved a good result. According to our single-patient experience, in patients of MOGCT with limited disease burden and no other effective systematic treatment available, salvage surgery may be considered. But when there was an elevated $\beta$-HCG level, the desperation surgery seemed less successful. Gene sequencing and target therapy may provide a tentative treatment for patients with refractory MOGCT, but the efficacy still needs to be verified.

\section{Consent}

Written informed consent was obtained from the patient for publication of this case report and any accompanying images.
Consent was obtained using the standardized informed consent forms of the participating institutions. The project and consent process were approved by the ethics committee of Peking Union Medical College Hospital, Beijing.

\section{Acknowledgments}

This work was supported by the Chinese Academy of Medical Sciences Initiative for Innovative Medicine (CAMS-2017I2M-1-002). We gratefully thank Professor Lina Guo and Yan You for their help in reviewing the pathology and providing pathological images. We are also grateful for the help provided by Scientific Precision Company on interpretation of genetic testing.

\section{Author contributions}

Conceived and designed the study: YW, J-XY, KS. Data acquisition: YW, MY, D-YC. Data analysis and interpretation: J-XY, MY. Writing - original draft: YW, MY. Writing - review \& editing: YW, J-XY, D-YC, KS. All authors have approved the final version to be published and agreed to be accountable for all aspects of the work in ensuring that questions related to the accuracy or integrity of any part of the work.

\section{Disclosure}

The authors report no conflicts of interest in this work.

\section{References}

1. Brown J, Friedlander M, Backes FJ, et al. Gynecologic Cancer Intergroup (GCIG) consensus review for ovarian germ cell tumors. Int $J$ Gynecol Cancer. 2014;24(9 Suppl 3):S48-S54.

2. Zhao Q, Yang J, Cao D, et al. Tailored therapy and long-term surveillance of malignant germ cell tumors in the female genital system: 10-year experience. J Gynecol Oncol. 2016;27(3):e26.

3. Smith HO, Berwick M, Verschraegen CF, et al. Incidence and survival rates for female malignant germ cell tumors. Obstet Gynecol. 2006; 107(5):1075-1085.

4. Bidus MA, Elkas JC, Rose GS. Germ cell, stromal, and other ovarian tumors. In: DiSaia PJ, Creasman WT, Mannel RS, McMeekin DS, Mutch DG, editors. Clinical Gynecologic Oncology. New York: Elsevier; 2012:290.e7-313.e7.

5. Gershenson DM. Management of ovarian germ cell tumors. J Clin Oncol. 2007;25(20):2938-2943.

6. Motzer RJ, Mazumdar M, Sheinfeld J, et al. Sequential dose-intensive paclitaxel, ifosfamide, carboplatin, and etoposide salvage therapy for germ cell tumor patients. J Clin Oncol. 2000;18(6):1173-1180.

7. Hoei-Hansen CE, Kraggerud SM, Abeler VM, Kaern J, Rajpert-de Meyts E, Lothe RA. Ovarian dysgerminomas are characterised by frequent KIT mutations and abundant expression of pluripotency markers. Mol Cancer. 2007;6:12.

8. Taylor-Weiner A,ZackT,O'DonnellE, etal. Genomic evolutionand chemoresistance in germ-cell tumours. Nature. 2016;540(7631):114-118.

9. Zou Y, Deng W, Wang F, etal. A novel somatic MAPK1 mutation in primary ovarian mixed germ cell tumors. Oncol Rep. 2016;35(2):725-730.

10. Werness BA, Ramus SJ, Whittemore AS, et al. Primary ovarian dysgerminoma in a patient with a germline BRCA1 mutation. Int $J$ Gynecol Pathol. 2000;19(4):390-394. 
11. Hamel N, Wong N, Alpert L, Galvez M, Foulkes WD. Mixed ovarian germ cell tumor in a BRCA2 mutation carrier. Int J Gynecol Pathol. 2007;26(2):160-164.

12. Cheng L, Roth LM, Zhang S, et al. KIT gene mutation and amplification in dysgerminoma of the ovary. Cancer. 2011;117(10):2096-2103.

13. Hersmus R, Stoop H, van de Geijn GJ, et al. Prevalence of c-KIT mutations in gonadoblastoma and dysgerminomas of patients with disorders of sex development (DSD) and ovarian dysgerminomas. PLoS One. 2012;7(8):e43952.

14. Witkowski L, Mattina J, Schönberger S, et al. DICER1 hotspot mutations in non-epithelial gonadal tumours. Br J Cancer. 2013;109(10): 2744-2750.

15. Hsu WH, Yang JC, Mok TS, Loong HH. Overview of current systemic management of EGFR-mutant NSCLC. Ann Oncol. 2018;29(Suppl 1): i3-i9.

16. Nishimura T, Nakamura K, Yamashita S, Ikeda S, Kigure K, Minegishi T. Effect of the molecular targeted drug, erlotinib, against endometrial cancer expressing high levels of epidermal growth factor receptor. BMC Cancer. 2015;15:957.

17. Chong CR, Jänne PA. The quest to overcome resistance to EGFRtargeted therapies in cancer. Nat Med. 2013;19(11):1389-1400.

18. Shaaban AM, Rezvani M, Elsayes KM, et al. Ovarian malignant germ cell tumors: cellular classification and clinical and imaging features. Radiographics. 2014;34(3):777-801.

19. Lai CH, Chang TC, Hsueh S, et al. Outcome and prognostic factors in ovarian germ cell malignancies. Gynecol Oncol. 2005;96(3):784-791.

20. Murugaesu N, Schmid P, Dancey G, et al. Malignant ovarian germ cell tumors: identification of novel prognostic markers and long-term outcome after multimodality treatment. J Clin Oncol. 2006;24(30): 4862-4866.

21. Ishiguro T, Yoshida Y, Tenzaki T, Ohshima M, Suzuki H. AFP in yolk sac tumor and solid teratoma of the ovary: significance of postoperative serum AFP. Cancer. 1981;48(11):2480-2484.

22. Ueda G, Abe Y, Yoshida M, Fujiwara T. Embryonal carcinoma of the ovary: a six-year survival. Int J Gynaecol Obstet. 1990;31(3): 287-292.

23. Chan JK, Tewari KS, Waller S, et al. The influence of conservative surgical practices for malignant ovarian germ cell tumors. $J$ Surg Oncol. 2008;98(2):111-116.

24. Ghalleb M, Bouzaiene H, Slim S, et al. Fertility-sparing surgery in advanced stage malignant ovarian germ cell tumor: a case report. J Med Case Rep. 2017;11(1):350.

25. Lu Y, Yang J, Cao D, et al. Role of neoadjuvant chemotherapy in the management of advanced ovarian yolk sac tumor. Gynecol Oncol. 2014; 134(1):78-83.

26. Gershenson DM, Morris M, Cangir A, et al. Treatment of malignant germ cell tumors of the ovary with bleomycin, etoposide, and cisplatin. J Clin Oncol. 1990;8(4):715-720.

27. Williams S, Blessing JA, Liao SY, Ball H, Hanjani P. Adjuvant therapy of ovarian germ cell tumors with cisplatin, etoposide, and bleomycin: a trial of the Gynecologic Oncology Group. J Clin Oncol. 1994;12(4): 701-706.

28. Kondagunta GV, Bacik J, Donadio A, et al. Combination of paclitaxel, ifosfamide, and cisplatin is an effective second-line therapy for patients with relapsed testicular germ cell tumors. J Clin Oncol. 2005;23(27): $6549-6555$

OncoTargets and Therapy

\section{Publish your work in this journal}

OncoTargets and Therapy is an international, peer-reviewed, open access journal focusing on the pathological basis of all cancers, potential targets for therapy and treatment protocols employed to improve the management of cancer patients. The journal also focuses on the impact of management programs and new therapeutic agents and protocols on
29. Lee SC, Kim KH, Kim SH, Lee NS, Park HS, Won JH. Mixed testicular germ cell tumor presenting as metastatic pure choriocarcinoma involving multiple lung metastases that was effectively treated with high-dose chemotherapy. Cancer Res Treat. 2009;41(4):229-232.

30. Pagliaro LC. Role of High-Dose Chemotherapy With Autologous Stem-Cell Rescue in Men With Previously Treated Germ Cell Tumors. J Clin Oncol. 2017;35(10):1036-1040.

31. Einhorn LH, Williams SD, Chamness A, Brames MJ, Perkins SM, Abonour R. High-dose chemotherapy and stem-cell rescue for metastatic germ-cell tumors. N Engl J Med. 2007;357(4):340-348.

32. Reddy Ammakkanavar N, Matei D, Abonour R, Einhorn LH. High-dose chemotherapy for recurrent ovarian germ cell tumors. J Clin Oncol. 2015;33(2):226-227.

33. de Giorgi U, Richard S, Badoglio M, et al. Salvage high-dose chemotherapy in female patients with relapsed/refractory germ-cell tumors: a retrospective analysis of the European Group for Blood and Marrow Transplantation (EBMT). Ann Oncol. 2017;28(8):1910-1916.

34. Oing C, Seidel C, Bokemeyer C. Therapeutic approaches for refractory germ cell cancer. Expert Rev Anticancer Ther. 2018;18(4):389-397.

35. Eastham JA, Wilson TG, Russell C, Ahlering TE, Skinner DG. Surgical resection in patients with nonseminomatous germ cell tumor who fail to normalize serum tumor markers after chemotherapy. Urology. 1994;43(1):74-80.

36. Murphy BR, Breeden ES, Donohue JP, et al. Surgical salvage of chemorefractory germ cell tumors. J Clin Oncol. 1993;11(2):324-329.

37. Albers P, Ganz A, Hannig E, Miersch WD, Müller SC. Salvage surgery of chemorefractory germ cell tumors with elevated tumor markers. J Urol. 2000;164(2):381-384.

38. Adra N, Einhorn LH, Althouse SK, et al. Phase II trial of pembrolizumab in patients with platinum refractory germ-cell tumors: a Hoosier Cancer Research Network Study GU14-206. Ann Oncol. 2018;29(1): 209-214.

39. Milewska M, Cremona M, Morgan C, et al. Development of a personalized therapeutic strategy for ERBB-gene-mutated cancers. Ther Adv Med Oncol. 2018;10:1758834017746040.

40. Wilken JA, Badri T, Cross S, et al. EGFR/HER-targeted therapeutics in ovarian cancer. Future Med Chem. 2012;4(4):447-469.

41. Mishra R, Hanker AB, Garrett JT. Genomic alterations of ERBB receptors in cancer: clinical implications. Oncotarget. 2017;8(69): 114371-114392.

42. Konecny GE, Pegram MD, Venkatesan N, et al. Activity of the dual kinase inhibitor lapatinib (GW572016) against HER-2-overexpressing and trastuzumab-treated breast cancer cells. Cancer Res. 2006;66(3): $1630-1639$.

43. Westphal M, Maire CL, Lamszus K. EGFR as a Target for Glioblastoma Treatment: An Unfulfilled Promise. CNS Drugs. 2017;31(9): 723-735.

44. Lee JK, Shin JY, Kim S, et al. Primary resistance to epidermal growth factor receptor (EGFR) tyrosine kinase inhibitors (TKIs) in patients with non-small-cell lung cancer harboring TKI-sensitive EGFR mutations: an exploratory study. Ann Oncol. 2013;24(8):2080-2087.

\section{Dovepress}

patient perspectives such as quality of life, adherence and satisfaction. The manuscript management system is completely online and includes a very quick and fair peer-review system, which is all easy to use. Visit http://www.dovepress.com/testimonials.php to read real quotes from published authors. 